\title{
Polar decomposition of complexified quaternions and octonions
}

\author{
Stephen J. Sangwine and Eckhard Hitzer
}

\begin{abstract}
We present a hitherto unknown polar representation of complexified quaternions (also known as biquaternions), also applicable to complexified octonions. The complexified quaternion is factored into the product of two exponentials, one trigonometric or circular, and one hyperbolic. The trigonometric exponential is a real quaternion, the hyperbolic exponential has a real scalar part and imaginary vector part. This factorisation is shown to be isomorphic to the polar decomposition of linear algebra.
\end{abstract}

Mathematics Subject Classification (2010). Primary 11R52; Secondary 15A23.

Keywords. biquaternion, quaternion, polar decomposition.

\section{Introduction}

A complex number may be expressed in polar form using the famous formula $e^{i \theta}=\cos \theta+i \sin \theta$ due to Euler. The polar form of a quaternion [13, p 56] is an important representation because it generalises Euler's formula to cases where the square root of -1 is a quaternion. That quaternions could be expressed in polar form was already clear to Hamilton in 1843 [6, Eqn: (s)] because he stated that, like complex numbers, they could be written in terms of a cosine and a sine of a single angle.

The polar form of biquaternions or complexified quaternions ${ }^{1}$ is a more recent discovery based on the roots of -1 in the biquaternions [9]. Several variant polar forms were set out in [11]. However, all of these previously known polar forms are based on a single exponential with a root of -1 . De Leo and Rodrigues [1] discussed polar forms of biquaternions and described a

This is a post-peer-review, pre-copyedit version of an article published in Advances in Applied Clifford Algebras. The final authenticated version is available online at: http: //dx.doi.org/10.1007/s00006-020-1048-y.

${ }^{1}$ The terms biquaternion and complexified quaternion are synonymous here and mean a quaternion with coefficients in $\mathbb{C}$. 
single polar form containing the product of two exponentials. However, the first of the exponentials was complex, and was therefore just representing a 'complex modulus' for the biquaternion. Both exponentials in their polar form were based on roots of -1 . Dorst and Valkenburg [2] worked out a polar decomposition of rotors in the conformal geometric algebra $\mathrm{Cl}(4,1)$, thus showing that polar decompositions can exist in hypercomplex algebras.

In this paper, we show that it is possible to factorise a unit biquaternion into a polar form as the product of two exponentials: one a quaternion exponential containing a root of -1 and a real angle, and the other a biquaternion exponential containing a root of +1 and again a real angle. The latter is a hyperbolic exponential based on hyperbolic cosine and sine functions, as we show in Lemma 1. The more general case of a biquaternion which is not of unit norm requires that we consider the complex semi-norm of the biquaternion, which can of course be done by fairly trivially including a third (complex) exponential with a real scalar modulus.

The factorisation is developed in this paper using direct algebraic manipulation of the biquaternion. However, it is also shown that this factorisation is equivalent (in fact isomorphic) to the polar decomposition [4, §4.2.10] or $[7, \S 13.3-4]$ of linear algebra which factorises a matrix into the product of a non-negative Hermitian matrix and a unitary matrix. Thus if we represent the biquaternion using a $4 \times 4$ complex matrix based on the usual matrix representation of quaternions [14], then the polar decomposition of the matrix yields the matrix representation of the two factors of the biquaternion, a result which has been verified computationally using [12]. This is why we call the decomposition/factorisation presented in this paper the polar decompo-

sition of a complexified quaternion. In section 6 we discuss the existence of the decomposition for complexified octonions, which follows exactly the same reasoning and algebra, and therefore does not need to be explicit. We also discuss the lack of a matrix representation of the polar decomposition in the octonion case due to the lack of a matrix representation of the full octonion product.

\section{Notation}

We use notation consistent with two earlier papers on biquaternions $[10,11]$ and also make use of some results and concepts from these papers.

We write full quaternions (i.e., those with non-zero vector part) as ordinary variables, thus $q$; pure quaternions with zero scalar part as bold Greek variables, thus $\boldsymbol{\alpha}$; matrices in uppercase roman font thus: A. A quaternion in Cartesian form is $q=w+x \boldsymbol{i}+y \boldsymbol{j}+z \boldsymbol{k}$, where $\boldsymbol{i}, \boldsymbol{j}, \boldsymbol{k}$ are the usual quaternion basis elements. We use $I$ for the complex root of -1 in order to distinguish it from the quaternion $\boldsymbol{i}$. The notation $\mathrm{S}(q)=w$ denotes the scalar part of a quaternion, and $\mathbf{V}(q)=x \boldsymbol{i}+y \boldsymbol{j}+z \boldsymbol{k}$ denotes the vector part. Quaternion conjugate (negation of the vector part) is denoted by an overbar: 
$\bar{q}=w-x \boldsymbol{i}-y \boldsymbol{j}-z \boldsymbol{k}=\mathrm{S}(q)-\mathbf{V}(q)$. Complex conjugation is indicated by a superscript star: $q^{\star}=w^{\star}+x^{\star} \boldsymbol{i}+y^{\star} \boldsymbol{j}+z^{\star} \boldsymbol{k}$.

The inner product of two quaternions is denoted $\langle p, q\rangle[13, \S 2.5]$ and can be computed as the sum of the products of corresponding coefficients of $p$ and $q$. This is a geometric scalar product in 4-space equivalent to treating the four coefficients of the quaternion as components of a 4-space vector. The norm of a quaternion is the sum of the squares of the coefficients: $\|q\|=$ $w^{2}+x^{2}+y^{2}+z^{2}$, and is also given by $\langle q, q\rangle$. The modulus is the square root of the norm: $|q|=\sqrt{\|q\|}$. When the coefficients are complex (as they are in biquaternions), the norm may be complex, and is strictly referred to as a semi-norm $[11, \S 3]$.

\section{Existence and computation of the polar factorisation}

We require the following result, which although straightforward, is not commonly listed in mathematical reference works in the full form given here. The first and third cases, but not the middle case, may be found in [3, Equations (2.3) and (2.25)]. Although we require here no more than $\theta \in \mathbb{R}$, the results are also valid for $\theta \in \mathbb{C}$.

Lemma 1. Let $\boldsymbol{\nu}$ be a hypercomplex ${ }^{2}$ root of $-1,0$, or +1 , that is either: $|\boldsymbol{\nu}|=1$ and $\boldsymbol{\nu}^{2}= \pm 1$, or $\boldsymbol{\nu}^{2}=0$ (in this case $|\boldsymbol{\nu}|=0$ ). Then the Euler formula has the following more general form:

$$
e^{\boldsymbol{\nu} \theta}= \begin{cases}\cos \theta+\boldsymbol{\nu} \sin \theta, & \boldsymbol{\nu}^{2}=-1 \\ 1+\boldsymbol{\nu} \theta, & \boldsymbol{\nu}^{2}=0 \\ \cosh \theta+\boldsymbol{\nu} \sinh \theta, & \boldsymbol{\nu}^{2}=+1\end{cases}
$$

Proof. All three cases follow easily from the series expansions of the exponential function and the trigonometric and hyperbolic functions (see, for example Korn and Korn [7, E-7(1-4)]), and the stated properties of $\boldsymbol{\nu}^{2}$.

We also require a second result which we have not been able to find in the literature (a related result is of course very well-known: that the quotient of two unit pure quaternions is a full quaternion, and if the two unit pure quaternions are orthogonal, the quotient is a unit pure quaternion). The result is a special case of the more general rotation in 4-space.

Lemma 2. The quotient of two orthogonal full quaternions is a pure quaternion.

Proof. Let $m$ and $n$ be unit full quaternions which are orthogonal. Thus $\mathrm{S}(m \bar{n})=\langle m, n\rangle=0$ by [8, Proposition 10.8]. $\mathrm{S}(m \bar{n})=0$ implies that

$$
m \bar{n}=\mathbf{V}(m \bar{n})
$$

\footnotetext{
${ }^{2}$ The term hypercomplex means a generalization of the complex numbers based on roots of \pm 1 , and with dimension $2,4,8$, etc. It is of course possible for this formula to work for other types of root than hypercomplex numbers (for example, matrices).
} 
The quotient of the two quaternions is $m \bar{n} /\|n\|$. Hence we have shown that the quotient of $m$ and $n$ is a pure quaternion.

Remark: it follows from the fact that the quaternions are a division algebra that if $m$ and $n$ are orthogonal and are both of unit modulus, their quotient will be a unit pure quaternion.

We consider unit biquaternions in what follows, with unit (and therefore real) semi-norm $[11, \S 3.3]$. (The semi-norm is a complex generalisation of the norm of a quaternion, computed by the same method - the square root of the sum of the squares of the four complex coefficients.) Since the seminorm is complex, it can be represented in the usual polar form with a real modulus and a real angle. In Corollary 1, we deal with the case of a general biquaternion with non-unit semi-norm, and show that the complex semi-norm simply scales the result.

Theorem 1. An arbitrary biquaternion $q$, with unit semi-norm, may be factored as follows:

$$
q=e^{\boldsymbol{\alpha} \theta_{t}} e^{I \boldsymbol{\beta} \theta_{h}}
$$

where $\boldsymbol{\alpha}$ and $\boldsymbol{\beta}$ are pure real unit quaternions (and therefore $\boldsymbol{\alpha}^{2}=\boldsymbol{\beta}^{2}=-1$ ); $I \boldsymbol{\beta}$ is a pure imaginary unit biquaternion (and therefore $(I \boldsymbol{\beta})^{2}=+1$ ). The two angles $\theta_{t}$ and $\theta_{h}$ are real, the subscripts standing for trigonometric and hyperbolic respectively.

In what follows we distinguish between the factors by referring to them as the trigonometric $\left(e^{\boldsymbol{\alpha} \theta_{t}}\right)$ and hyperbolic $\left(e^{I \boldsymbol{\beta} \theta_{h}}\right)$ factors. An alternative factorisation exists with the factors reversed, since they do not commute, in general. The trigonometric factor is real, since $\boldsymbol{\alpha}$ and $\theta_{t}$ are real, and it is invariant to the ordering of the factors. The hyperbolic factor has a rather special structure: it has a real scalar part, and an imaginary vector part. This can be seen by inspection of the third case in equation (1). The scalar part of the hyperbolic factor is the modulus of the real part of $q$. The imaginary part of the hyperbolic factor can be obtained by left division of the trigonometric factor into the imaginary part of $q$, but it is also possible to divide by the trigonometric factor on the left of $q$ to obtain the entire hyperbolic factor.

Any biquaternion (with the exception of divisors of zero), may be normalised to unit form by dividing by its semi-norm. We deal with the special case of a divisor of zero later in the paper: the factorisation works even in this case, but one of the factors will be a divisor of zero.

Proof. The proof assumes a general biquaternion with unit semi-norm, from which by a series of algebraic steps, we obtain the right-hand side of equation 2 .

Represent an arbitrary biquaternion $q$ with unit semi-norm in the complex form II of [11, Table 2], that is, in the form of a complex number with real quaternion coefficients:

$$
q=q_{r}+I q_{i}
$$


where $q_{r}$ and $q_{i}$ are real quaternions. Then write the real and imaginary parts in the usual quaternion polar form:

$$
q=\left|q_{r}\right| e^{\boldsymbol{\mu}_{r} \theta_{r}}+I\left|q_{i}\right| e^{\boldsymbol{\mu}_{i} \theta_{i}}
$$

where $\boldsymbol{\mu}_{r}$ and $\boldsymbol{\mu}_{i}$ are unit pure quaternion roots of -1 , and $\theta_{r}$ and $\theta_{i}$ are real.

We now use two consequences of the fact that $\|q\|$ is real (because $\|q\|=$ 1). These are given in [10, Lemma 1]. Firstly, we have that $\left\|q_{r}\right\|-\left\|q_{i}\right\|=1$, or equivalently, $\left|q_{r}\right|^{2}-\left|q_{i}\right|^{2}=1$, which requires that $\left|q_{r}\right|=\cosh \psi$ and $\left|q_{i}\right|=$ $\sinh \psi$ for some $\psi$ to be determined. Secondly, $\left\langle q_{r}, q_{i}\right\rangle=0$ which implies that $e^{\boldsymbol{\mu}_{r} \theta_{r}} \perp e^{\boldsymbol{\mu}_{i} \theta_{i}}$. Two orthogonal unit quaternions have a quotient that is a unit pure quaternion. This follows from Lemma 2. Therefore we can introduce a unit pure quaternion $\boldsymbol{\nu}$, with $\boldsymbol{\nu}^{2}=-1$, and write $e^{\boldsymbol{\mu}_{i} \theta_{i}}=e^{\boldsymbol{\mu}_{r} \theta_{r}} \boldsymbol{\nu}$. Making use of both these consequences, we can write:

$$
q=\cosh \psi e^{\boldsymbol{\mu}_{r} \theta_{r}}+I \sinh \psi e^{\boldsymbol{\mu}_{r} \theta_{r}} \boldsymbol{\nu}
$$

which can be factored as follows:

$$
q=e^{\boldsymbol{\mu}_{r} \theta_{r}}(\cosh \psi+I \boldsymbol{\nu} \sinh \psi)
$$

or, by Lemma 1

$$
q=e^{\boldsymbol{\mu}_{r} \theta_{r}} e^{I \boldsymbol{\nu} \psi}
$$

which matches the theorem as stated with the obvious identifications between variables.

A simple algorithm to compute the factorisation follows directly from Equation 6 - the trigonometric factor is obtainable from the real part of the biquaternion, since $q_{r}=e^{\boldsymbol{\alpha} \theta_{t}} \cosh \theta_{h}$ (using the variables in Theorem 1). Normalising this removes the hyperbolic cosine to give a unit quaternion which is the trigonometric factor. The hyperbolic factor can then be obtained by division (using multiplication by the inverse of the trigonometric factor, the inverse being the quaternion conjugate, since the trigonometric factor is a unit quaternion). This is the algorithm implemented in the Quaternion Toolbox for MATLAB ${ }^{T M}[12$, function: polar], for both biquaternions and complexified octonions, which has been used to verify the results in this paper.

Corollary 1. The result in Theorem 1 generalizes to the case of a biquaternion $p$ which does not have unit modulus (square root of the semi-norm), as follows:

$$
p=|p| q=|p| e^{\boldsymbol{\alpha} \theta_{t}} e^{I \boldsymbol{\beta} \theta_{h}}, \quad \text { where } \quad|p| \in \mathbb{C}, \text { assuming }|p| \neq 0,
$$

that is, $|p|$ scales the result (and commutes with the unit biquaternion $q$, since $|p|$ is complex). (We consider the case where $|p|=0$ in $\S 4$.)

Proof. The semi-norm of a biquaternion $p$ may be expressed by $\|p\|=p \bar{p}$, and the modulus by the square root of this result $[11, \S 3.3]$. Dividing $p$ by its modulus gives a biquaternion of unit modulus, $q=p / \sqrt{p \bar{p}}$ which factorises as in Theorem 1 . That $q$ has unit modulus may be shown by computing its 
norm using the same formula as just stated, noting that $\sqrt{p \bar{p}}$ is complex and therefore unaffected by taking a quaternion conjugate:

$$
\|q\|=q \bar{q}=\frac{p}{\sqrt{p \bar{p}}} \frac{\bar{p}}{\sqrt{p \bar{p}}}=\frac{p \bar{p}}{p \bar{p}}=1
$$

from which it follows that $|q|=1$.

To conclude this section we present a worked numerical example. We take $q=1+(1+1 I) \boldsymbol{i}+(1-1 I) \boldsymbol{j}$, which has $\|q\|=1$. The real part is $q_{r}=1+1 \boldsymbol{i}+1 \boldsymbol{j}$ which has $\left\|q_{r}\right\|=3$. The orthogonal imaginary part is $q_{i}=\boldsymbol{i}-\boldsymbol{j}$, which has $\left\|q_{i}\right\|=2$, so $\|q\|=\left\|q_{r}\right\|-\left\|q_{i}\right\|=3-2=1$. Hence, the trigonometric factor is $e^{\boldsymbol{\alpha} \theta_{t}}=(1+1 \boldsymbol{i}+1 \boldsymbol{j}) / \sqrt{3}$ from which $\boldsymbol{\alpha}=(\boldsymbol{i}+\boldsymbol{j}) / \sqrt{2}$ and $\tan \theta_{t}=\sqrt{2}$ may be obtained by the usual quaternion formulae.

To obtain the hyperbolic factor we need to divide $q$ on the left (or right if we desire the alternative factorisation) by the trigonometric factor. Taking the conjugate of the trigonometric factor we have:

$$
\begin{aligned}
e^{I \boldsymbol{\beta} \theta_{h}}=e^{-\boldsymbol{\alpha} \theta_{t}} q & =\frac{1}{\sqrt{3}}(1-1 \boldsymbol{i}-1 \boldsymbol{j})(1+(1+1 I) \boldsymbol{i}+(1-1 I) \boldsymbol{j}) \\
& =\frac{1}{\sqrt{3}}(3+1 I \boldsymbol{i}-1 I \boldsymbol{j}+2 I \boldsymbol{k})
\end{aligned}
$$

from which it is possible to find $\boldsymbol{\beta}=\frac{1}{\sqrt{3}}((\boldsymbol{i}-\boldsymbol{j}) / \sqrt{2}+\sqrt{2} \boldsymbol{k})$, and $\cosh \theta_{h}=$ $\sqrt{3}$. That these results are correct may be verified numerically using [12] by substitution into (2). Note that in this case, $\boldsymbol{\alpha}$ and $\boldsymbol{\beta}$ are orthogonal ${ }^{3}$, but this is not a general property, as can be verified easily by factoring a random example numerically.

\section{Degenerate cases and divisors of zero}

In the previous section we dealt with biquaternions of unit norm/semi-norm, and biquaternions with a complex but non-zero semi-norm. In fact the factorization can be defined even in the case of divisors of zero (with some provisos as we see below), and for degenerate cases. By degenerate we mean cases where some part of the biquaternion is zero, for example the scalar part, or the imaginary part. Although these were covered implicitly above, the ramifications were not, and that is what we present next.

\subsection{Degenerate cases}

As we have seen in Corollary 1 , the factorisation of a biquaternion $p$ with non-unit norm was written as:

$$
p=|p| q=|p| e^{\boldsymbol{\alpha} \theta_{t}} e^{I \boldsymbol{\beta} \theta_{h}}
$$

where $|p|$ may be complex. Degenerate cases occur where $p$ is wholly real or wholly imaginary:

\footnotetext{
${ }^{3}$ We thank one of the referees for pointing out the orthogonality of $\boldsymbol{\alpha}$ and $\boldsymbol{\beta}$ in the example.
} 
- If $p$ is a real quaternion, the decomposition is trivial: $|p|$ is real, the trigonometric factor $e^{\boldsymbol{\alpha} \theta_{t}}$ is a unit version of $p$, and the hyperbolic factor $e^{I \boldsymbol{\beta} \theta_{h}}$ must be unity. The polar decomposition therefore reduces to the well-known polar form of a real quaternion.

- If $p$ is an imaginary quaternion (a biquaternion with zero real part), the decomposition is again trivial: $|p|$ is imaginary, and after normalising the biquaternion, $p /|p|$ is real and of unit modulus. Therefore the trigonometric factor $e^{\boldsymbol{\alpha} \theta_{t}}=p /|p|$, and again the hyperbolic factor must be unity.

\subsection{Divisors of zero}

If $p$ is a divisor of zero, there must be non-zero real and imaginary parts. We cannot extract the semi-norm or modulus (since the semi-norm of a divisor of zero is zero) $[10, \S 3]$. However, we can extract the real part of $p$ and normalise it. This gives us a trigonometric factor of unit modulus, and of course a real modulus which we may choose to keep as a separate factor or to combine with the hyperbolic factor. Dividing $p$ by the trigonometric factor yields the hyperbolic factor which will be a divisor of zero.

A numerical example will make this clearer. Let

$$
p=\frac{1}{2}(1+1 \boldsymbol{i}+1 I \boldsymbol{j}-1 I \boldsymbol{k}) .
$$

This is an idempotent, meaning that $p^{2}=p$, and it has a vanishing seminorm: $\|p\|=0$. The real part of $p$ is $p_{r}=\frac{1}{2}(1+1 \boldsymbol{i})$, with $\left|p_{r}\right|=\frac{1}{\sqrt{2}}$. Normalising this gives $e^{\boldsymbol{\alpha} \theta_{t}}=\frac{1}{\sqrt{2}}(1+1 \boldsymbol{i})$ from which $\boldsymbol{\alpha}=\boldsymbol{i}$ and $\theta_{t}=$ $\pi / 4$. We now choose to divide out of $p$ the real part, including its modulus, to obtain the hyperbolic factor. Therefore $e^{I \boldsymbol{\beta} \theta_{h}}=\frac{1}{\sqrt{2}}(1-1 \boldsymbol{i}) q=$ $\frac{1}{\sqrt{2}}(1-1 I \boldsymbol{k})$ which is a divisor of zero. Dividing this result by $\sqrt{2}$ gives the idempotent value $\frac{1}{2}(1-1 I \boldsymbol{k})$. Examination of the hyperbolic factor reveals that it is not possible to find $\theta_{h}$ since there is no value of $\theta_{h}$ for which $\cosh \theta_{h}=\sinh \theta_{h}$ other than at infinity. However, it is easy to verify that $p=\sqrt{2}\left(\frac{1}{\sqrt{2}}(1+1 \boldsymbol{i})\right)\left(\frac{1}{2}(1-1 I \boldsymbol{k})\right)$, the three factors being, from left to right, a real scale factor, the real trigonometric factor (which may also be written as $e^{i \pi / 4}$, and the hyperbolic (idempotent) factor.

\section{Relation to the polar decomposition of linear algebra}

We have used the name polar decomposition in this paper for the result in Theorem 1 for good reason: it is isomorphic to the polar decomposition of matrices in linear algebra $[4, \S 4.2 .10]$ or $[7, \S 13.3-4(3)]$. In order to demonstrate the isomorphism for the case of biquaternions we need two concepts. Firstly, quaternions have a $4 \times 4$ matrix representation, as given, for example, by Ward $[13, \S 2.8]$. A quaternion $q=w+x \boldsymbol{i}+y \boldsymbol{j}+z \boldsymbol{k}$ is represented in 
matrix form as $^{4}$ :

$$
\left(\begin{array}{rrrr}
w & -x & -y & -z \\
x & w & -z & y \\
y & z & w & -x \\
z & -y & x & w
\end{array}\right)
$$

This means we can represent a biquaternion using a $4 \times 4$ complex matrix and the product of two biquaternions by the matrix product of their equivalent matrices. Secondly, we need the equivalences between the biquaternion operations of quaternion conjugate and complex conjugate, and the corresponding operations on the matrix representation. These are as follows:

- the quaternion conjugate (the negation of the vector part) corresponds to the matrix transpose as can be seen by inspection of the matrix given above;

- the complex conjugate (of the four complex coefficients) of the biquaternion corresponds to the complex conjugation of all the matrix elements.

The Hermitian transpose of linear algebra, which both transposes the matrix and conjugates the complex elements of the matrix, corresponds to the application of both types of conjugation: the quaternion conjugate and the complex conjugate of the coefficients of the biquaternion.

Now we note that in the linear algebra case of the polar decomposition, the matrix corresponding to our hyperbolic factor is obtained by the following expression $[7, \S 13.3-4(3)]$, where $\mathrm{A}=\mathrm{QU}$ is the matrix to be factorised $^{5}$ : $\mathrm{Q}=\sqrt{\mathrm{A}^{\dagger} \mathrm{A}}$, where the square root denotes a matrix square root, and $\dagger$ denotes a Hermitian transpose. Given the equivalences noted above, in order to show that the factorisation presented in this paper is isomorphic to the linear algebra polar decomposition, it is sufficient to show that the hyperbolic factor (denoted here by $q_{h}=e^{I \boldsymbol{\beta} \theta_{h}}$ ) can be obtained using the same formula (with equivalent operations substituted for the matrix operations). We do this by substitution of the result in Theorem 1 into the formula just stated, replacing the Hermitian transpose of linear algebra by the double conjugation of quaternion and complex conjugation. Therefore we need to demonstrate that:

$$
q_{h}=\sqrt{\overline{\left(e^{\boldsymbol{\alpha} \theta_{t}} e^{I \boldsymbol{\beta} \theta_{h}}\right)^{\star}} e^{\boldsymbol{\alpha} \theta_{t}} e^{I \boldsymbol{\beta} \theta_{h}}}
$$

The complex conjugate has no effect on the trigonometric factor $e^{\boldsymbol{\alpha} \theta_{t}}$ because $\boldsymbol{\alpha}$ and $\theta$ are real, but it does change the sign of $I$ in the hyperbolic factor $e^{I \boldsymbol{\beta} \theta_{h}}$ (recall that $\boldsymbol{\beta}$ is also real, so it does not change $\boldsymbol{\beta}$ ):

$$
q_{h}=\sqrt{\left(e^{\boldsymbol{\alpha} \theta_{t}} e^{-I \boldsymbol{\beta} \theta_{h}}\right)} e^{\boldsymbol{\alpha} \theta_{t}} e^{I \boldsymbol{\beta} \theta_{h}}
$$

The quaternion conjugate applied to a product reverses the order of the terms and conjugates each ${ }^{6}: \overline{p q}=\bar{q} \bar{p}$. Further, the quaternion conjugate of

\footnotetext{
${ }^{4}$ The transpose of this matrix is also valid, one or other must be chosen by convention.

${ }^{5}$ An alternative with the Hermitian transpose on the right is also possible, corresponding to the alternative ordering of the factors in Theorem 1.

${ }^{6}$ This statement has the following equivalent in matrix form: $(P Q)^{T}=Q^{T} P^{T}$.
} 
an exponential changes the sign of the exponent. Therefore, applying the conjugate to the bracketed exponentials, we have:

$$
q_{h}=\sqrt{e^{I \boldsymbol{\beta} \theta_{h}} e^{-\boldsymbol{\alpha} \theta_{t}} e^{\boldsymbol{\alpha} \theta_{t}} e^{I \boldsymbol{\beta} \theta_{h}}}
$$

Finally, the middle pair of trigonometric exponentials cancel, and the pair of hyperbolic exponentials reduce under the square root to:

$$
q_{h}=e^{I \boldsymbol{\beta} \theta_{h}}
$$

which is the hyperbolic factor in Theorem 1. As noted in Section 3, it is simpler and faster to compute the hyperbolic factor by dividing out the trigonometric factor rather than by using the expression $\sqrt{\overline{q^{\star}} q}$.

Although the polar decomposition presented in this paper is isomorphic to the polar decomposition of linear algebra, it is worth pointing out that the algorithm for computing the polar decomposition in this paper is inevitably considerably faster than the linear algebra case, as is always the case with quaternion computations: the matrix representation has 4-fold redundancy, and requires both more memory, and a greater number of numerical operations; it is also not guaranteed that the special form of the matrix will be preserved accurately over a series of computational steps.

We now return to the numerical example with which we concluded $\S 3$, but this time in its isomorphic matrix form. Recall that we took $q=1+(1+$ $1 I) \boldsymbol{i}+(1-1 I) \boldsymbol{j}$. In isomorphic matrix form this biquaternion is:

$$
Q=\left(\begin{array}{cccc}
1 & -1-1 I & -1+1 I & 0 \\
1+1 I & 1 & 0 & 1-1 I \\
1-1 I & 0 & 1 & -1-1 I \\
0 & -1+1 I & 1+1 I & 1
\end{array}\right)
$$

The matrix representation of the trigonometric factor can be found directly from $Q$ by taking the real part of $Q$ and dividing by its 2 -norm ${ }^{7}$, which gives:

$$
T=\frac{1}{\sqrt{3}}\left(\begin{array}{rrrr}
1 & -1 & -1 & 0 \\
1 & 1 & 0 & 1 \\
1 & 0 & 1 & -1 \\
0 & -1 & 1 & 1
\end{array}\right)
$$

which is the matrix representation of the trigonometric factor found in $\S 3$.

The matrix representation of the hyperbolic factor can be obtained by $H=T^{-1} Q$ or by $H=\sqrt{Q^{\dagger} Q}$, either of which gives the following result, which can be seen on inspection to be isomorphic to the hyperbolic factor given in $\S 3$ :

$$
H=\frac{1}{\sqrt{3}}\left(\begin{array}{cccc}
3 & -1 I & 1 I & -2 I \\
1 I & 3 & -2 I & -1 I \\
-1 I & 2 I & 3 & -1 I \\
2 I & 1 I & 1 I & 3
\end{array}\right)
$$

\footnotetext{
${ }^{7}$ This method is specific to the biquaternion case, and is not applicable to the polar decomposition of matrices in general.
} 
TABLE 1. Octonion multiplication table, as implemented in [12].

\begin{tabular}{l|rrrrrrrr} 
& 1 & $\boldsymbol{i}$ & $\boldsymbol{j}$ & $\boldsymbol{k}$ & $\boldsymbol{l}$ & $\boldsymbol{m}$ & $\boldsymbol{n}$ & $\boldsymbol{o}$ \\
\hline 1 & 1 & $\boldsymbol{i}$ & $\boldsymbol{j}$ & $\boldsymbol{k}$ & $\boldsymbol{l}$ & $\boldsymbol{m}$ & $\boldsymbol{n}$ & $\boldsymbol{o}$ \\
$\boldsymbol{i}$ & $\boldsymbol{i}$ & -1 & $\boldsymbol{k}$ & $-\boldsymbol{j}$ & $\boldsymbol{m}$ & $-\boldsymbol{l}$ & $-\boldsymbol{o}$ & $\boldsymbol{n}$ \\
$\boldsymbol{j}$ & $\boldsymbol{j}$ & $-\boldsymbol{k}$ & -1 & $\boldsymbol{i}$ & $\boldsymbol{n}$ & $\boldsymbol{o}$ & $-\boldsymbol{l}$ & $-\boldsymbol{m}$ \\
$\boldsymbol{k}$ & $\boldsymbol{k}$ & $\boldsymbol{j}$ & $-\boldsymbol{i}$ & -1 & $\boldsymbol{o}$ & $-\boldsymbol{n}$ & $\boldsymbol{m}$ & $-\boldsymbol{l}$ \\
$\boldsymbol{l}$ & $\boldsymbol{l}$ & $-\boldsymbol{m}$ & $-\boldsymbol{n}$ & $-\boldsymbol{O}$ & -1 & $\boldsymbol{i}$ & $\boldsymbol{j}$ & $\boldsymbol{k}$ \\
$\boldsymbol{m}$ & $\boldsymbol{m}$ & $\boldsymbol{l}$ & $-\boldsymbol{o}$ & $\boldsymbol{n}$ & $-\boldsymbol{i}$ & -1 & $-\boldsymbol{k}$ & $\boldsymbol{j}$ \\
$\boldsymbol{n}$ & $\boldsymbol{n}$ & $\boldsymbol{o}$ & $\boldsymbol{l}$ & $-\boldsymbol{m}$ & $-\boldsymbol{j}$ & $\boldsymbol{k}$ & -1 & $-\boldsymbol{i}$ \\
$\boldsymbol{o}$ & $\boldsymbol{o}$ & $-\boldsymbol{n}$ & $\boldsymbol{m}$ & $\boldsymbol{l}$ & $-\boldsymbol{k}$ & $-\boldsymbol{j}$ & $\boldsymbol{i}$ & -1
\end{tabular}

These matrix results may be verified using $[12]^{8}$.

\section{Octonion case}

Without formal proof, the decomposition presented here works also for octonions with complex coefficients (complexified octonions). This is because all of the algebraic steps presented in the proof of Theorem 1 are valid for octonions, and at no point does a product of more than two octonions occur, which would cause problems with associativity.

It is well-known that the octonions cannot have a matrix representation - because the matrix product is associative, the multiplication of octonions cannot be represented by multiplication of matrices. It is clearly possible to represent the product of two octonions by a matrix-vector product, representing the left octonion by a matrix and the right octonion by a column vector. Therefore, although the polar decomposition as presented in Theorem 1 will work, the correspondence with the polar decomposition of linear algebra, as presented in Section 5, cannot, because it is not possible to construct matrices that will represent the octonion factors.

We present an example, and as in the case of the examples presented earlier for biquaternions, this example may be verified numerically using [12, function: polar]. At this point we introduce additional notation for the octonion basis elements, using $\boldsymbol{i}, \boldsymbol{j}, \boldsymbol{k}$ as before for biquaternions, and $\boldsymbol{l}, \boldsymbol{m}, \boldsymbol{n}, \boldsymbol{o}$ for the further four needed to complete the 7 -dimensional octonion basis ${ }^{9}$. Since there are multiple possible multiplication tables for the octonions, we state in Table 1 the multiplication table being used ${ }^{10}$.

\footnotetext{
${ }^{8}$ The matrix representation of a biquaternion $q$ can be computed using the function call adjoint (q, 'real').

${ }^{9}$ This is a slight but common abuse of notation - the quaternion and octonion $\boldsymbol{i}, \boldsymbol{j}, \boldsymbol{k}$ should be regarded as distinct.

${ }^{10}$ This choice follows from the way the octonions are implemented in [12] as a pair of quaternions using the Cayley-Dickson construction:
}$$
(w+x \boldsymbol{i}+y \boldsymbol{j}+z \boldsymbol{k})+(a+b \boldsymbol{i}+c \boldsymbol{j}+d \boldsymbol{k}) \boldsymbol{l}=w+x \boldsymbol{i}+y \boldsymbol{j}+z \boldsymbol{k}+a \boldsymbol{l}+b \boldsymbol{m}+c \boldsymbol{n}+d \boldsymbol{o} .
$$ 
Let $p=(1+2 \boldsymbol{i}+3 \boldsymbol{j}+4 \boldsymbol{k}+5 \boldsymbol{l}+6 \boldsymbol{m}+7 \boldsymbol{n}+8 \boldsymbol{o})+I(8+7 \boldsymbol{i}+6 \boldsymbol{j}+5 \boldsymbol{k}+$ $4 \boldsymbol{l}+3 \boldsymbol{m}+2 \boldsymbol{n}+1 \boldsymbol{o})$. The polar factorisation gives the following results (computed numerically, and then converted to the form given using the MATLAB ${ }^{\text {TM }}$ symbolic toolbox):

- Semi-norm: $\|p\|=240 I$ and modulus: $|p|=2 \sqrt{30}(1+1 I)$;

- Trigonometric factor: $\frac{1}{2 \sqrt{2}}(1+1 \boldsymbol{i}+1 \boldsymbol{j}+1 \boldsymbol{k}+1 \boldsymbol{l}+1 \boldsymbol{m}+1 \boldsymbol{n}+1 \boldsymbol{o})$;

- Hyperbolic factor: $\frac{1}{2 \sqrt{15}}(9-I(4 \boldsymbol{l}-1 \boldsymbol{m}-2 \boldsymbol{o}))$.

From these values we can obtain:

- $\boldsymbol{\alpha}=\frac{1}{\sqrt{7}}(1 \boldsymbol{i}+1 \boldsymbol{j}+1 \boldsymbol{k}+1 \boldsymbol{l}+1 \boldsymbol{m}+1 \boldsymbol{n}+1 \boldsymbol{o})$,

- $\tan \theta_{t}=\sqrt{7}$,

- $\boldsymbol{\beta}=\frac{-1}{\sqrt{21}}(4 \boldsymbol{l}+1 \boldsymbol{m}+2 \boldsymbol{o})$

- $\tanh \theta_{h}=\frac{\sqrt{7}}{3 \sqrt{3}}$

Substitution of these values into (2) will confirm their correctness using [12].

\section{Conclusion}

In this paper we have presented a hitherto unknown polar factorisation of complexified quaternions, and proved its equivalence to the polar decomposition of linear algebra. We have shown how to compute the factorisation. We have also shown less formally, that the factorisation is also applicable to complexified octonions, and presented an argument as to why there cannot be an equivalence with the polar decomposition of linear algebra.

\section{Acknowledgement}

We thank the two referees who reviewed this paper very thoroughly and made some excellent suggestions for minor improvements, which have, we believe, improved the paper considerably.

\section{References}

[1] Stefano de Leo and Waldyr A. Rodrigues, Jr. Quaternionic electron theory: Geometry, algebra, and Dirac's spinors. International Journal of Theoretical Physics, 37(6):1707-1720, June 1998.

[2] Leo Dorst and Robert Valkenburg. Square root and logarithm of rotors in 3D conformal geometric algebra using polar decomposition. In L. Dorst and J. Lasenby, editors, Guide to Geometric Algebra in Practice, chapter 5, pages 81-104. Springer-Verlag, London, 2011.

[3] Catoni F., Boccaletti D., Cannata R., Catoni V., and Zampetti P. Hyperbolic numbers. In Geometry of Minkowski Space-Time, SpringerBriefs in Physics, chapter 2. Springer, Berlin, Heidelberg, 2011.

[4] Gene H. Golub and Charles F. van Loan. Matrix Computations. Johns Hopkins studies in the Mathematical Sciences. The Johns Hopkins University Press, Baltimore and London, third edition, 1996. 
[5] W. R. Hamilton. On a new species of imaginary quantities connected with the theory of quaternions. Proceedings of the Royal Irish Academy, 2:424-434, 1844 .

[6] W. R. Hamilton. On a new species of imaginary quantities connected with the theory of quaternions. In H. Halberstam and R. E. Ingram, editors, The Mathematical Papers of Sir William Rowan Hamilton, volume III Algebra, chapter 5, pages 111-116. Cambridge University Press, Cambridge, 1967. First published as [5].

[7] Granino A. Korn and Theresa M. Korn. Mathematical Handbook for Scientists and Engineers. Dover, Mineola, NY, 2000. Unabridged republication of McGraw-Hill edition, 1968.

[8] I. R. Porteous. Topological Geometry. Cambridge University Press, Cambridge, second edition, 1981.

[9] S. J. Sangwine. Biquaternion (complexified quaternion) roots of -1 . Advances in Applied Clifford Algebras, 16(1):63-68, June 2006.

[10] S. J. Sangwine and Daniel Alfsmann. Determination of the biquaternion divisors of zero, including the idempotents and nilpotents. Advances in Applied Clifford Algebras, 20(2):401-410, May 2010. Published online 9 January 2010.

[11] S. J. Sangwine, T. A. Ell, and N. Le Bihan. Fundamental representations and algebraic properties of biquaternions or complexified quaternions. Advances in Applied Clifford Algebras, 21(3):607-636, September 2011.

[12] Stephen J. Sangwine and Nicolas Le Bihan. Quaternion Toolbox for Matlab@, version 2 with support for octonions. [Online], 2013. Software library available at: http://qtfm. sourceforge.net/.

[13] J. P. Ward. Quaternions and Cayley Numbers: Algebra and Applications, volume 403 of Mathematics and Its Applications. Kluwer, Dordrecht, 1997.

[14] F. Z. Zhang. Quaternions and matrices of quaternions. Linear Algebra and its Applications, 251:21-57, January 1997.

Stephen J. Sangwine

School of Computer Science and Electronic Engineering, University of Essex, Wivenhoe Park, Colchester, CO4 3SQ, United Kingdom.

e-mail: sjs@essex.ac.uk

Eckhard Hitzer

International Christian University, Osawa 3-10-2, Mitaka-shi 181-8585, Tokyo, Japan. e-mail: hitzer@icu.ac.jp 